\title{
The Taipan snake venom time: a new test for lupus anticoagulant
}

\author{
A M Rooney, T McNally, I J Mackie, S J Machin
}

\begin{abstract}
Aims-To develop a specific test for lupus anticoagulant activity with reduced sensitivity to coagulation factor deficiency that would be suitable for analysis of plasmas from patients receiving oral anticoagulants.
\end{abstract}

Methods-A coagulation test based on the Taipan snake venom time (TSVT) with a platelet neutralisation procedure (PNP) was developed and compared with dilute Russell's viper venom time (DRVVT). The TSVT was used to test plasmas from patients receiving oral anticoagulant or heparin with mild liver dysfunction and with documented lupus anticoagulant.

Results-The optimised conditions for the TSVT were established and a reference range was determined in normal healthy subjects. Results were considered positive for lupus anticoagulant if the ratio was $\geq 1 \cdot 1$ and was reduced by $\geq 10 \%$ or to $<1 \cdot 1$ in the PNP. In 43 samples from patients receiving oral anticoagulants there was no correlation between level of anticoagulation and TSVT, and only seven samples had increased TSVTs. Of these, five corrected on mixing with normal plasma and two gave equivocal results. The patients with mild liver dysfunction all had normal TSVTs. The TSVT in plasmas from patients receiving heparin correlated with the heparin concentrations (as measured by the APTT, $\mathbf{r}^{2}$ $=0.81$ ). Some anticoagulated plasmas showed correction in the PNP and were regarded as false positive. Fourteen of 17 patients known to have lupus anticoagulant (on the basis of DRVVT results) were also positive by the TSVT; two of the remaining three were borderline and one was negative.

Conclusions-The TSVT showed satisfactory intra-assay precision and reasonable sensitivity to lupus anticoagulant, compared with the DRVVT. The TSVT was influenced by the presence of heparin but was not sensitive to the effects of oral anticoagulant. Like other lupus anticoagulant tests, it does not seem to havea $100 \%$ detection rate, but this may be due to the presence of lupus anticoagulant subtypes with distinct activities or the requirement of cofactors other than prothrombin or $\beta_{2}$ glycoprotein-I.

(F Clin Pathol 1994;47:497-501)

The lupus anticoagulant prolongs the clotting times of phospholipid dependent in vitro coagulation tests. ${ }^{1}$ This is due to the presence of plasma immunoglobulins (usually IgG, IgM, or a mixture of both) which bind to the anionic phospholipids that normally support haemostatic reactions. Lupus anticoagulant was first described in patients with systemic lupus erythematosus (SLE), ${ }^{2}$ but lupus anticoagulants have since been demonstrated in a variety of clinical conditions. The presence of lupus anticoagulant has been associated with thrombosis and recurrent fetal loss, ${ }^{34}$ and these associations have established the importance of laboratory diagnosis of lupus anticoagulant. There is still considerable controversy about the most appropriate method for detecting lupus anticoagulant. Current recommendations in the United Kingdom suggest that laboratories should screen plasma and then use at least one more specific confirmatory test. ${ }^{5}$ The activated partial thromboplastin time (APTT) is the most frequently used screening test for lupus anticoagulant ${ }^{6}$ and confirmatory tests include the dilute Russell's viper venom time (DRVVT) and the kaolin clotting time (KCT). ${ }^{5}$ The use of mixing studies with normal plasma and correction procedures with lysed washed platelets ${ }^{7}$ or high concentrations of phospholipid ${ }^{8}$ have been a further development in laboratory diagnosis of lupus anticoagulant. Regardless of which method is used, care must be taken to ensure that laboratory procedures are standardised. ${ }^{5}$

Most patients referred for investigation of lupus anticoagulant have had previous thrombotic episodes and are consequently receiving anticoagulants. However, all of the standard tests used for lupus anti-coagulant are affected by oral anticoagulant therapy. Mixtures of normal plasma and test plasma may correct a factor deficiency without neutralising the lupus anti-coagulant effect, but the sensitivity of the testis reduced and the current recommenda-tions advise repeat testing after discontinuing oral anticoagulants. ${ }^{5}$ In many cases this cannot be considered because of the increased risk of thrombosis. We have explored the used of a reagent prepared from the venom of the Taipan snake (Oxyuranus scutellatus) that directly activates prothrombin in the presence of phospholipid and calcium ions, ${ }^{9}$ for the identification of lupus anticoagulant in patients receiving oral anticoagulants.

Methods

Venous blood was collected into a one-tenth 
volume of $0.106 \mathrm{M}$ tri-sodium citrate and centrifuged at $2000 \times g$ for 15 minutes to prepare plasma. Platelet poor plasma (PPP) was prepared by recentrifuging the plasma at $2000 \times g$ for a further 15 minutes. All samples were stored in aliquots at $-70^{\circ} \mathrm{C}$.

Patients were considered positive for lupus anticoagulant according to the British Society for Haematology guidelines on testing for the lupus anticoagulant ${ }^{5}$ - that is, on the basis of the results of the APTT and DRVVT - with appropriate mixing and correction tests. APTT was measured using a micronised silica reagent (Instrumentation Laboratories Ltd, Warrington, Cheshire). Thrombin time (TT) was measured using bovine thrombin and prothrombin time (PT) using phenolised rabbit brain thromboplastin (Diagnostic Reagents Ltd, Thame, Oxford). The DRVVT was measured using "Bell and Alton" phospholipid reagent and Russell's viper venom (RVV) (Diagnostic Reagents Ltd). Freeze-thawed, washed platelets for the platelet neutralisation procedure (PNP) of the DRVVT and Taipan snake venom test (TSVT) were prepared from normal citrated platelet rich plasma (PRP) using a washing buffer containing inhibitors of platelet activation. ${ }^{10}$ The washed platelets
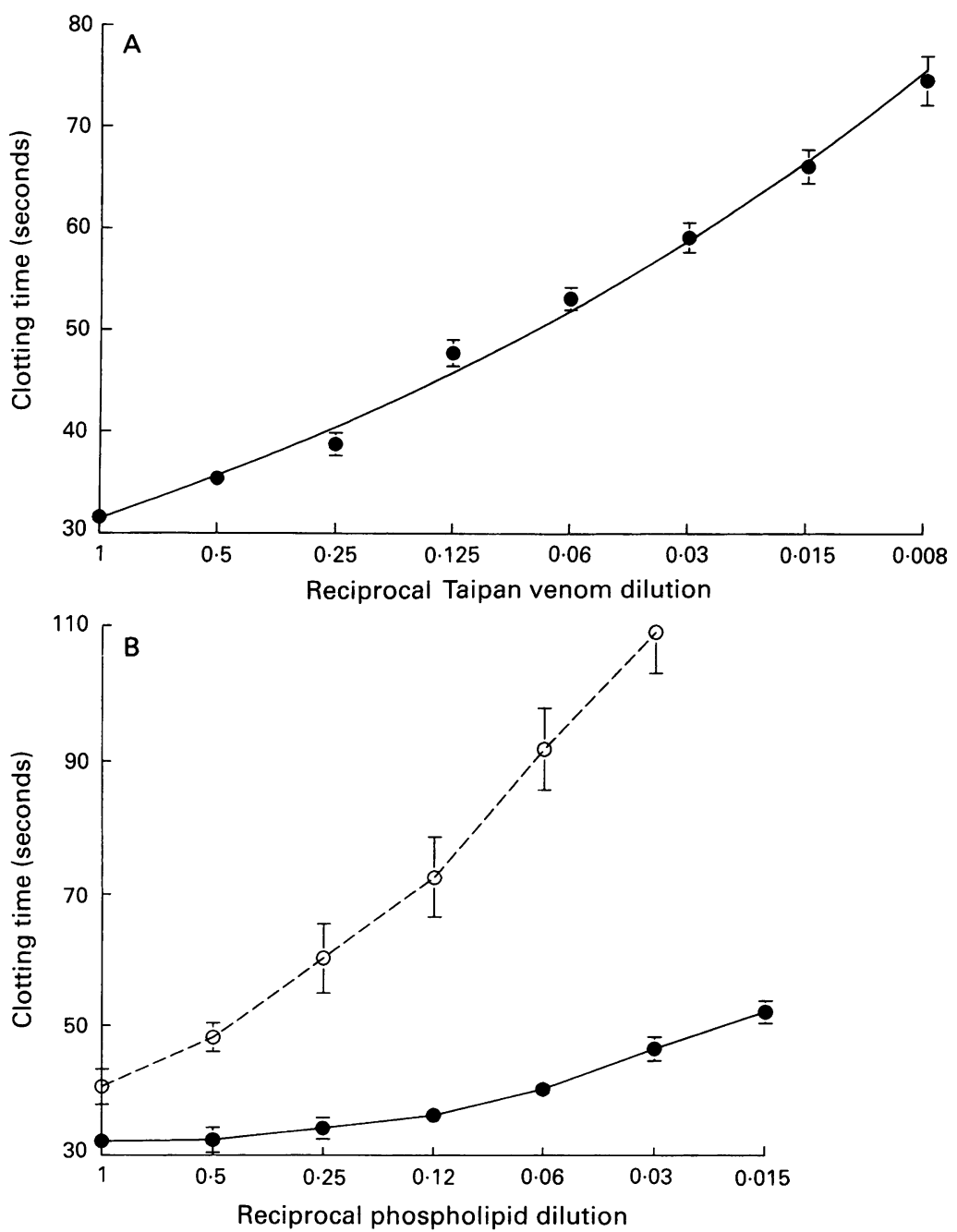

Figure 1 (A) The effect of Taipan snake venom concentration on the TSVT (B) The effect of phospholipid concentration on the TSVT of a normal (- $\longrightarrow$ ) and well characterised LA plasma (O-- $)$. were finally suspended in buffer without inhibitors at a count of $400 \times 10^{9} / 1$ and frozen at $-70^{\circ} \mathrm{C}$ before thawing and freezing in aliquots at $-70^{\circ} \mathrm{C}$.

The following conditions for the TSVT were defined on the basis of results of preliminary optimisation experiments performed using normal PPP and a well characterised strong lupus anticoagulant PPP. "Bell and Alton" phospholipid was diluted 1 in 4 in $0.05 \mathrm{M}$ imidazole, $0.1 \mathrm{M} \mathrm{NaCl}, \mathrm{pH} \mathrm{7.3}$. Taipan snake venom (TSV) (Diagnostic reagents Ltd) was reconstituted according to manufacturer's instructions and then diluted with an equal volume of $0.025 \mathrm{M}$ calcium chloride. $(100 \mu \mathrm{l})$ phospholipid and $100 \mu \mathrm{l}$ normal control or test PPP were mixed and incubated in a $75 \times 10 \mathrm{~mm}$ glass tube at $37^{\circ} \mathrm{C}$ for 30 seconds. TSV $(100 \mu \mathrm{l})$ were added and the clotting time measured. The test was then repeated with the platelet neutralisation procedure (PNP), substituting washed lysed platelets for phospholipid. A ratio of test and control clotting times was calculated and if the test:control ratio with phospholipid was $\geq 1 \cdot 1$ the test was repeated using a 1:1 mixture of normal control and test plasma (NP mix). A positive result for lupus anticoagulant in the TSVT was defined as a ratio of $\geq 1 \cdot 1$ with correction to $\leq 1 \cdot 1$ or by $10 \%$ or more with the PNP. Two commercial lyophilised washed platelet preparations, Platelet Extract Reagent (Alpha Laboratories, Eastleigh, Hampshire, England) and Platelet Neutralisation Reagent (Quatro Biosystems, Manchester, Lancashire England), were also tested in the TSVT.

The optimised TSVT was used to study healthy normal subjects, patients receiving anticoagulants, patients with liver dysfunction, and patients referred for investigation for thrombophilia.

\section{Results}

When the Taipan venom concentration was decreased at a constant phospholipid concentration, there was a progressive increase in the clotting time of normal plasma (fig 1A). A 1 in 2 dilution of Taipan venom was selected for further experiments to confer sensitivity while maintaining convenient clotting times. Decreasing the phospholipid concentration at a constant venom concentration produced progressively increasing clotting times with both normal plasma and a well characterised lupus anticoagulant plasma (fig 1B). A 1 in 4 dilution of phospholipid in imidazole buffer was selected for use as this produced minimal prolongation of the clotting time of normal plasma, while permitting clear distinction between normal and lupus anticoagulant positive clotting times. Dilution of the washed platelets from a suspension of $\approx 400 \times 10^{9} / 1$ resulted in increased clotting times with both normal and lupus anticoagulant plasmas (not shown). A 1 in 4 dilution of this reagent was selected for further experiments to maintain convenient clotting times. Two commercial PNP reagents were also tested and had 
Table 1 Results of stability studies on TSV reagent

\begin{tabular}{llllll}
\hline \multirow{4}{*}{$\begin{array}{l}\text { Time after } \\
\text { reconstitution of } \\
\text { TSV (hours) }\end{array}$} & \multicolumn{3}{l}{$\begin{array}{l}\text { TSVT ratio of lupus anticoagulant } \\
\text { positive plasma }\end{array}$} \\
\cline { 2 - 3 } & TSV stored on ice & & \multicolumn{2}{l}{ Freeze-thawed TSV } \\
\cline { 2 - 3 } \cline { 5 - 6 } TSVT & PNP & & TSVT & PNP \\
\hline 0 & 1.35 & 1.07 & & 1.35 & 1.07 \\
1 & 1.35 & 1.05 & & 1.28 & 1.15 \\
2 & 1.40 & 1.07 & & 1.28 & 1.09 \\
4 & 1.34 & 1.16 & & 1.27 & 1.05 \\
6 & 1.28 & 1.09 & & 1.30 & 1.04 \\
8 & 1.27 & 1.10 & & 1.35 & 1.02 \\
\hline
\end{tabular}

comparable performance to the "in house" reagent.

The stability of the Taipan reagent was assessed by measuring the TSVT of a lupus anticoagulant plasma at varying time intervals over an eight hour period using Taipan venom reagent that was stored on ice during this period or repeatedly freeze-thawed. The Taipan venom reagent remained stable on ice and was unaffected by repeated freeze-thawing (table 1). Three different batches of TSV were tested and demonstrated a similar potency and gave a similar pattern of results with normal and lupus anticoagulant positive plasmas.

Intra-assay precision of the optimised TSVT was assessed by assaying normal plasma and a well characterised lupus anticoagulant plasma 20 times. The intra-assay coefficients of variation (CV) of ratios of the TSVT and PNP of the lupus anticoagulant plasma were determined to be $4 \%$ and $3 \%$, respectively. The interassay precision was assessed by assaying a separate aliquot of an lupus anticoagulant plasma on nine consecutive runs over a six week period and was determined to be $13 \%$ and $7 \cdot 3 \%$ for the TSVT and PNP, respectively. Although a certain degree of interassay variation was apparent in both the normal and lupus anticoagulant positive plasmas, the lupus anticoagulant plasma was correctly identified on each occasion.

The optimised method was used to mea-

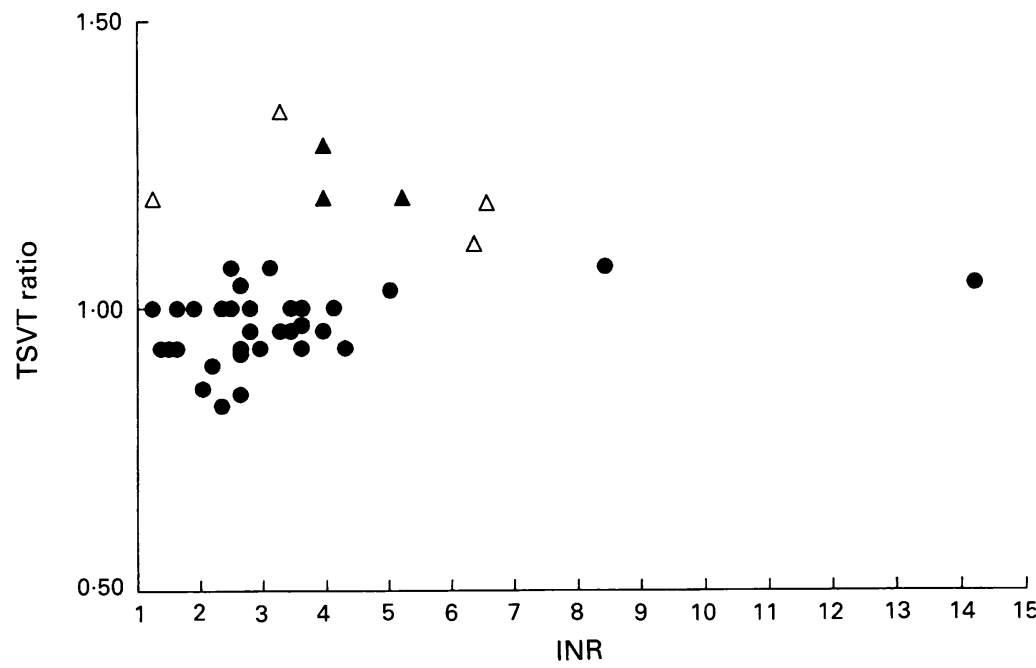

Figure 2 TSVT results of plasmas from patients receiving oral anticoagulant treatment normal TSVT, $\triangle$ increased TSVT, $\triangle$ indicates increased TSVT results of plasmas from one patient.
Table 2 Results of correction studies performed on warfarin plasmas with abnormal TSVT results

\begin{tabular}{llllll}
\hline $\begin{array}{l}\text { Case } \\
\text { No }\end{array}$ & INR & TSVT & PNP & NP mix & Result \\
\hline 1 & 1.24 & 1.19 & 1.00 & 1.07 & ?Positive \\
2 & 3.27 & 1.34 & 1.08 & 1.14 & ?Positive \\
3 & 3.95 & 1.28 & 1.18 & 1.07 & Negative \\
4 & 3.95 & 1.19 & 1.16 & ND & Negative \\
5 & 5.21 & 1.19 & 1.16 & 0.93 & Negative \\
6 & 6.36 & 1.11 & 1.29 & 0.96 & Negative \\
7 & 6.56 & 1.18 & 1.19 & 0.93 & Negative \\
\hline
\end{tabular}

sure the TSVT of 20 normal subjects. This group included 12 women and eight men and had a mean age of 30 years (range 21-56 years). The mean (SD) of the TSVT ratios were $0.97(0.05)$ and the reference range (mean 2SD) was therefore determined as 0.87-1.07.

Samples were collected from 43 patients receiving oral anticoagulants. The patient group included those starting treatment and those attending the oral anticoagulant clinic, in order to achieve a wide range of levels of anticoagulation. Some patients had international normalised ratio (INR) results below the therapeutic range $<2 \cdot 5$ ) and a small number had grossly increased INRs $(>4 \cdot 0)$. There was no correlation between INR and TSVT (fig 2). Thirty six of the patients receiving warfarin had normal TSVT ratios, irrespective of INR, and seven plasmas from five patients had increased TSVT ratios. Of the seven plasmas with increased TSVT ratios, two corrected by more than $10 \%$ or to $\leq 1 \cdot 1$ in the PNP. These plasmas also corrected to $<1 \cdot 1$ (case 1 ) and by more than $10 \%$ (case 2 ) in a 1:1 mix with normal plasma and so were considered as possible lupus anticoagulant positive plasmas (table 2). Five plasmas did not correct with PNP and of these, four were found to correct when a 1:1 with normal plasma was tested, indicating that prolongation of the TSVT was due to factor deficiency. The remaining plasma was not tested with a 1:1 mix with normal plasma but was from a

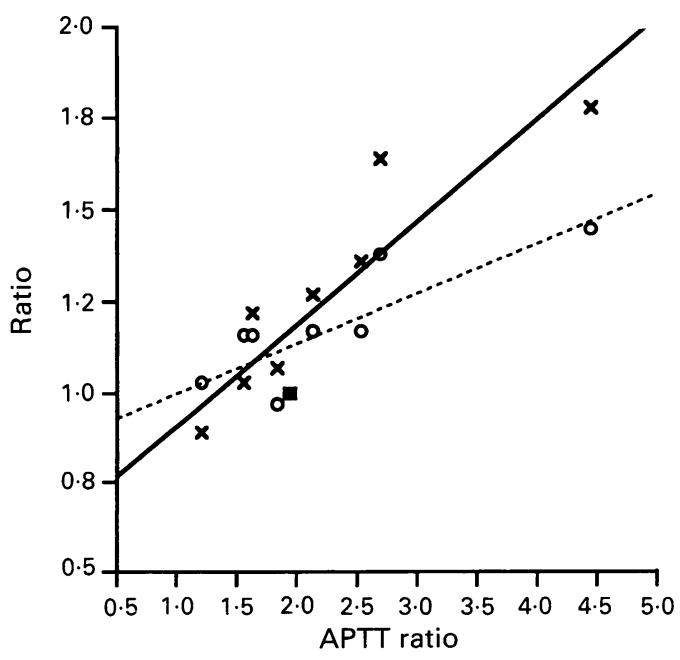

Figure 3 TSVT results of plasmas from patients receiving heparin $X-\ldots-\ldots T S V T$, O-- - PNP. 
Table 3 Median DRVVT and TSVT results of lupus anticoagulant positive patients

\begin{tabular}{lcccc}
\hline & DRVVT & DRVVT PNP & TSVT & TSVT PNP \\
\hline n= & 17 & 17 & 17 & 17 \\
Median & 1.84 & 1.50 & 1.86 & 1.31 \\
Minimum & 1.10 & 0.99 & 1.06 & 1.00 \\
Maximum & 5.60 & 2.84 & 2.71 & 1.74 \\
\hline
\end{tabular}

patient found to be negative for lupus anticoagulant on two other occasions.

Nine plasmas from seven patients receiving unfractionated heparin and selected at random were tested to examine the effect of heparin on the TSVT (fig 3). Heparin resulted in prolongation of the TSVT and a significant correlation was demonstrated between APTT ratio and TSVT $\left(r^{2}=0.81, p\right.$ $<0.001)$. Five patients had TSVT ratios of $\geq 1.1$ and in three of these the PNP corrected the TSVT ratio by more than $10 \%$, giving an apparent positive result, while the remaining two patients corrected by $<10 \%$.

Twelve patients with liver dysfunction (secondary to alcoholic liver disease, paracetamol overdose, or obstructive jaundice) with a mean INR of 1.52 (range $1 \cdot 12-2 \cdot 19$ ) and thrombin time of 15.5 (range 13.0-19.0) were tested. All patients had normal TSVT ratios (not shown).

We also investigated 17 patients who had been diagnosed as lupus anticoagulant positive on the basis of DRVVT results. The median DRVVT and TSVT results are shown in table 3. Fourteen patients were found to be positive for lupus anticoagulant by the TSVT. Of the remaining three, one had a normal TSVT (1.06), one had a borderline TSVT $(1.09)$ which corrected with the PNP (1.00), and one had a prolonged TSVT $(1 \cdot 23)$ but gave $<10 \%$ correction with the PNP $(1 \cdot 14)$.

We also tested 16 patients attending the thrombophilia clinic for investigation of previous thrombotic episodes who had abnormal DRVVTs but did not demonstrate the correction patterns typical of lupus anticoagulant. Ten of these patients had normal TSVT results. The remaining six patients all showed $>10 \%$ correction with PNP but also demonstrated $>10 \%$ correction in a $1: 1 \mathrm{mix}$ with normal plasma. Thus the TSVT allowed 10 of these patients to be classified as lupus anticoagulant negative but could not clarify the situation in the remainder.

\section{Discussion}

The clinical importance of antiphospholipid antibodies is becoming increasingly recognised and consequently lupus anticoagulant testing has become an essential routine procedure for haemostasis laboratories. Incidental findings of lupus anticoagulant are relatively rare and it is more usual for testing to be required after a previous unexplained thrombotic episode, often when the patient is receiving oral anticoagulants. Consequently it may be difficult to interpret results as the available tests are all dependent on adequate levels of several coagulation factors and are therefore affected by oral anticoagulants.

Phospholipid dependent prothrombin activating snake venoms are suitable candidate reagents for lupus anticoagulant test systems with reduced sensitivity to oral anticoagulants, as they rely only on adequate concentrations of prothrombin and fibrinogen and are not affected by plasma concentrations of factors IX and $\mathrm{X}$. The Taipan venom from Oxyuranus scutellatus contains at least one prothrombin activator of large molecular size. ${ }^{9}$ The protein apparently consists of a factor Xa-like catalytic subunit and factor Va-like cofactor unit which are potentiated by the presence of phospholipid and calcium ions. The products of venom catalysed prothrombin activation are thrombin and meizothrombin. ${ }^{11}$ Activators such as these that have no factor $\mathrm{Va}$ requirement have as yet only been identified in the venoms of Australian Elapidae. ${ }^{12}$

The action of lupus anticoagulant is universally agreed to centre on the inhibition of the role of phospholipid in haemostatic reactions. Therefore, the amount of phospholipid in a test system should be limited, as high phospholipid concentrations may mask the inhibitory effect of lupus anticoagulants. In the TSVT a 1 in 4 dilution of phospholipid resulted in good distinction between normal and lupus anticoagulant plasma clotting times, whereas higher dilutions resulted in very long clotting times, giving logistical problems for routine use and weaker clot formation, which made accurate end-point determination difficult. Confirmation of the presence of lupus anticoagulant requires correction of the clotting time on addition of lysed washed platelets or excess phospholipid, but no correction on mixing with normal plasma. ${ }^{513} \mathrm{~A} 1$ in 4 dilution of the stock suspension of washed platelets was selected for use and gave good correction $(>20 \%)$ of the TSVT ratio using a well characterised lupus anticoagulant plasma. Preparation of washed platelets is time consuming and so two commercial products designed for use in the PNP in the APTT or DRVVT were tested in the TSVT and performed well.

One of the disadvantages of the DRVVT is that the RVV is unstable and activity is reduced over time, even at $4^{\circ} \mathrm{C} .{ }^{14}$ The results of assays performed with TSV stored on ice over an eight hour period or repeatedly freezethawed suggest that TSV is stable, even in a diluted form. This may be due to the incorporation of albumin or other stabilisers by the manufacturer in the particular TSV reagent used. This may not be the case with other products and therefore TSV stability may vary according to source.

Prolongation of the TSVT was generally not related to the degree of oral anticoagulant treatment; the TSVT was normal in two patients with grossly raised INR values (8.4 and $14 \cdot 2$ ). Five anticoagulated patients did have prolonged TSVTs and of these, three corrected to $<1 \cdot 1$ with a $50 / 50$ mix with normal plasma, indicating that the prolongation 
was due to factor deficiency. These patients might have had more severely depressed prothrombin concentrations as a result of concomitant liver dysfunction. In the remaining two, the TSVT corrected in the PNP and so they must be considered as possible lupus anticoagulant positive patients.

A number of patients with mild liver dysfunction were tested by the TSVT. All patients had normal TSVT results, suggesting that the fibrinogen and vitamin $\mathrm{K}$ dependent coagulation factor abnormalities induced by this condition do not adversely affect the TSVT.

A correlation was shown between heparin treatment (as measured by the APTT) and TSVT. Although prolongation of the TSVT and PNP was demonstrated with increased heparin concentrations the PNP was less prolonged than the TSVT at the higher heparin values, giving a false positive effect. This is most likely attributable to the heparin neutralising activity of platelet factor 4 in the washed platelet preparation. Plasmas contaminated with heparin therefore cannot be reliably tested using the TSVT and testing should be repeated when heparin treatment has been stopped.

The efficacy of the TSVT in identifying lupus anticoagulant positive plasmas was assessed by testing known lupus anticoagulant positive plasmas. The patient group comprised patients with SLE and antiphospholipid syndrome and included some patients receiving oral anticoagulant treatment. The minimum and median values of the DRVVT and TSVT were comparable but the maximum level of the TSVT was much lower than that of the DRVVT. This difference is most likely a reflection of the reduced sensitivity of the TSVT to oral anticoagulants. The TSVT correctly identified all but one of the lupus anticoagulant positive plasmas. This plasma did not fulfil the lupus anticoagulant criteria of the TSVT but was identified as lupus anticoagulant positive using the DRVVT. The reason for this discrepancy is unclear; it is possible that the lupus anticoagulant may be part of a distinct subgroup with its main mode of action directed at a site other than the prothrombinase complex which forms the basis of the TSVT.

The TSVT was also used to test several plasmas which gave prolonged DRVVT results but did not give correction results typical of lupus anticoagulant. Most of these plasmas yielded normal TSVTs. Plasmas demonstrating prolonged TSVTs all showed correction with a 50/50 mix with normal plasma and correction with PNP. These results show that the TSVT is far less liable to false positive analysis than the DRVVT.
The TSVT can therefore be considered a suitable test for the diagnosis of lupus anticoagulant. The TSVT demonstrated satisfactory intra-assay precision and reasonable sensitivity compared with the DRVVT. It has the advantage of requiring only adequate fibrinogen and prothrombin concentrations and has reduced sensitivity to factor deficiency, an important consideration in the analysis of plasmas from patients receiving oral anticoagulants. An additional advantage is that the test is based on prothrombin, a relatively nonlabile coagulation factor, and as such may be more readily applied than the DRVVT to the assay of previously freeze-thawed plasmas. The test is easy to perform, readily adaptable for automated operation, and relatively inexpensive and is therefore suitable for use in most haemostasis laboratories.

T McNally was supported by a grant from the Arthritis and Rheumatism Council (No M0136).

1 Shapiro SS, Thiagarajan P. Lupus anticoagulants. In: Spaet TH, ed. Progress in thrombosis and haemostasis. Vo 6. New York: Grune \& Stratton, 1982:263-85.

2 Conley CL, Hartman RC. A haemorrhagic disorder caused by circulating anticoagulant in patients with disseminated lupus erythematosus. F Clin Invest 1952; 31:621-62.

3 Bowie WEJ, Thompson JH, Pascuzzi CA, Owen GA. Thrombosis in systemic lupus erythematosus despite circulating anticoagulants. F Lab Clin Med 1963;62: culating

4 Nilsson IM, Asted B, Hedner U, Berezin D. Interuterine death and circulating anticoagulant, 'antithromboplasdeath'. Acta Med Scand 1975;197:153-75.

5 The Lupus Anticoagulant Working Party of The British Society for Haematology. Guidelines on testing for the lupus anticoagulant. F Clin Pathol 1991;44:885-9.

6 The Lupus Anticoagulant Working Party of The British Society for Haematology. Detection of the lupus-like anticoagulant: current laboratory practice in The United Kingdom. F Clin Pathol 1990;43:73-5.

7 Triplett DA, Brandt JT, Kaczor D, Scaeffer J. Laboratory diagnosis of lupus inhibitors: A comparison of the tissue thromboplastin inhibition procedure with a new platelet neutralisation procedure. Am $f$ Clin Pathol 1983; 79:678-82.

8 Rosove $\mathrm{MH}$, Ismail $\mathrm{M}$, Kozoil BJ, Runge A, Kasper CK Lupus anticoagulants: Improved diagnosis with a kaolin clotting time using rabbit brain thromboplastin in standard and high concentrations. Blood 1986;68:472-8.

9 Speijer H, Govers-Reimslag JWP, Zwaal RFA, Rosing J Prothrombin activation by an activator from the venom of Oxyuranus scutellatus (Taipan snake). $\mathcal{F}$ Biol Chem 1986;261:13258-67.

10 Machin SJ, Mackie IJ. Platelet function tests. In: Chanarin I, ed. Laboratory haematology. An account of laboratory techniques. Edinburgh: Churchill Livingstone, 1989:382.

11 Walker FJ, Owen WG, Esmon CT. Characterisation of the prothrombin activator from the venom of Oxyuranus prothrombin activator from the venom of Oxyuranus 1980;19:1020-3.

12 Rosing J, Tans G. Inventory of exogenous prothrombin activators for the subcommittee on nomenclature of exogenous haemostatic factors of the scientific and standardisation committee of the International Society on Thrombosis and Haemostasis. Thromb Haemostas 1991; 65:627-30.

13 Exner T, Triplett DA, Taberner D, Machin SJ. Guidelines for testing and revised criteria for lupus anticoagulants, prepared for the subcommittee for the standardization of lupus anticoagulants of the International Society on Thrombosis and Haemostasis. Thromb Haemostas 1911; 65:320-2.

14 Sukhu K, Mackie IJ. Automation of the DRVVT. Med Lab Sci 1992;49:183-4. 